\title{
Mitteilungen aus der SGG, der SFGG und der SGAP
}

\section{Informations de la SSG, de la SPSG et de la SPPA}

\section{Übersicht}

Abschied und Dank

Fachgruppe Angewandte Gerontologie (FGAG)

Fachgruppe Physiotherapie (FGP)

Schweizerische Fachgesellschaft für

Gerontopsychologie (SFGP)

Arbeitsgruppe Gerontechnologie

Arbeitsgruppe Pflegewohnungen

Arbeitsgruppe Netzwerk Altersbeauftragte

Arbeitsgruppe Ethik und Spiritualität

Schweizerische Fachgesellschaft für Geriatrie (SFGG)

Schweizerische Gesellschaft für Alterspsychiatrie

und -psychotherapie (SGAP)

\section{Aperçu}

Au revoir et merci

Groupe des professionnels en gérontologie appliquée (GPGA)

Groupe spécialisé de physiothérapie en gériatrie

(GPG)

Société professionnelle suisse de gérontopsychologie (SPGP)

Groupe de travail Gérontechnologies

70

Groupe de travail «Appartements médicalisés»

Groupe de travail «Réseau des coordinateurs pour personnes âgées»

Groupe de travail Ethique et spiritualité

Société Professionnelle Suisse de Gériatrie (SPSG) 71

Société Suisse de Psychiatrie et Psychothérapie de la

Personne Agée (SPPA)

\section{Abschied und Dank \\ Liebe Mitglieder der SGG}

Mit dem Kongress 2016 geht meine Amtszeit zu Ende. Für Ihre Unterstützung der SGG im Rahmen Ihrer beruflichen und ehrenamtlichen Tätigkeit während meiner Amtszeit, aber hoffentlich auch darüber hinaus, möchte ich Ihnen, liebe Mitglieder, ganz herzlich danken. Ohne Ihren Beitrag, sei es im wissenschaftlichen oder im angewandten Feld der Gerontologie, kann die SGG keine Wirkung erzielen und zur Verbesserung der Lebenssituation der älteren Menschen in unserer Gesellschaft heute und morgen beitragen!

Ebenso gilt mein Dank Ihrem Vertrauen, das es mir ermöglicht hat, mich während vier Jahren aktiv für die Anliegen der Gerontologie und die Ziele der SGG einzusetzen. Es war eine sehr spannende und intensive Zeit, die mir über das Amtsbezogene hinaus viele persönliche Begegnungen ermöglicht hat. Diese werden sicher auch weiter Bestand haben und mich als Gerontologin und Privatperson bereichern.

Mein Dank gilt insbesondere auch dem ehrenamtlichen Engagement der in unseren diversen Fach- und Arbeitsgruppen engagierten Mitglieder: der Fachgruppe Angewandte Gerontologie (FGAG) mit ihren regionalen Foren,

der Fachgruppe Physiotherapie (FGP) mit ihren stets gut besuchten Weiterbildungsveranstaltungen sowie der Fachgesellschaft Gerontopsychologie (SFGP), die sich für altersgerechte Therapiemöglichkeiten einsetzt. Einen wichtigen Beitrag leisten auch unsere Arbeitsgruppen: die AG Ethik und Spiritualität mit der Erarbeitung der erfolgreichen Broschüre „Ethische Entscheidfindung in der ambulanten und stationären Langzeitpflege“, die AG Gerontechnologie, die sich aktiv in der Kongressgestaltung 2016 eingebracht hat, sowie die AG Pflegewohnungen, welche sich für neue Betreuungsformen stark macht.

Ganz besonders danken möchte ich auch allen Mitgliedern des Vorstandes der SGG für die persönlich sehr angenehme und vor allem konstruktive Zusammenarbeit. Sie haben mich mit ihren Fragen und Diskussionsbeiträgen immer wieder angeregt und manchmal durchaus auch gefordert, aber in jedem Fall die Entwicklung der SGG enorm befördert. Und last but not least wäre mein ganzes Engagement für die SGG nicht möglich gewesen, wenn ich nicht auf die ausserordentlich kompetente und konstruktive Unterstützung unsere Geschäftsstelle, namentlich von Monika Steiger und Beatrice Böhlen, hätte zählen dürfen!

Meinen ganz herzlichen Dank an Euch alle, ich durfte vier spannende Amtsjahre erleben und gemeinsam haben wir einiges erreicht! 
Mein Abschied ist jedoch nur ein "halber“, denn als Präsidentin past werde ich mich gerne weiter für die SGG engagieren und ihr so verbunden bleiben! Dem neuen Präsidium und Vorstand wünsche ich bereits heute alles Gute, viel Erfolg bei der weiteren Arbeit und vor allem genauso viel Befriedigung, wie ich sie erleben durfte!
Herzlich

Ihre

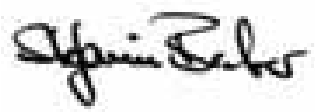

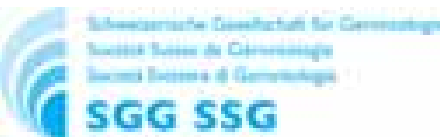

\section{Fachgruppe Angewandte Gerontologie (FGAG)}

\section{Weg frei für ein viertes FGAG-Forum Angewandte Gerontologie!}

Der Vorstand der Fachgruppe Angewandte Gerontologie FGAG hat an seiner Septembersitzung mit Freude von den Plänen für ein viertes regionales Forum für Angewandte Gerontologie Kenntnis genommen. Die Kerngruppe für das neue Forum in der Region Nordwestschweiz umfasst bereits vier Personen. Alle FGAG-Mitglieder aus der Region werden noch persönlich angeschrieben.

Die nächsten Termine der FGAG-Foren Angewandte Gerontologie in Bern, Luzern und Zürich stehen bereits fest:

Forum Angewandte Gerontologie Region Zürich/ Ostschweiz

- Dienstag, 26.1.2016, 18.00 Uhr

- Mittwoch, 1.6.2016, 18.00 Uhr

- Donnerstag, 29.9.2016, 18.00 Uhr
Forum Angewandte Gerontologie Region Bern

- Montag, 22. Februar 2016, 16.30 Uhr

- Montag, 13. Juni 2016, 16.30 Uhr

- Montag, 24. Oktober 2016, 16.30 Uhr

Forum Angewandte Gerontologie Region Zentralschweiz

- Donnerstag, 10. März 2016, 17.00 Uhr

- Donnerstag 12 Mai 2016, 17.00 Uhr

- Donnerstag, 15. September 2016, 17.00 Uhr

- Donnerstag, 1. Dezember 2016, 17.00 Uhr

NEU: Forum Angewandte Gerontologie Region Nordwestschweiz (Beide Basel, Aargau, Solothurn)

Die Daten werden auf www.sgg-ssg.ch/ FGAG veröffentlicht

Angaben über die jeweils vorgesehenen Themen und den Ort der Gesprächsveranstaltungen finden Sie unter www. sgg-ssg.ch (FGAG).

Für den FGAG-Vorstand, Stefan Spring

\section{Fachgruppe Physiotherapie in der Geriatrie (FPG)}

\section{Bilder sagen mehr als Worte}

Eines ihrer grossen Ziele fürs 2015 hat die FPG erreicht: Das Bilderarchiv ist erstellt. Über 100 Bilder zeigen eindrücklich, was wir PhysiotherapeutInnen alles bewegen können. Damit eine Therapie gelingen kann, braucht es eine vertrauensvolle Beziehung zwischen PatientIn und TherapeutIn. Dies und vieles mehr hat der Fotograf Bruno Wyss für uns eingefangen. Die Fotos können bald auf der SGG-Homepage (> Fachgruppen $>$ FPG) bestaunt werden. Für die Nutzung dieser Fotos ist seitens der FPG Ansprechperson: Frau Christine
Frey-Widmer, physio.frey@gmx.ch, (Tel G: 04349781 17). Anfragen können jederzeit an sie gestellt werden.

Ein weiterer Meilenstein wurde in der Zusammenarbeit mit der Fachhochschule Gesundheit in Winterthur (ZHAW) erreicht. Ab 2017 kann ein modular aufgebauter CAS in geriatrischer Physiotherapie absolviert werden. Am 27. August 2016 wird dieses neue Angebot näher vorgestellt im Rahmen eines Symposiums zum Thema: "Neue Wege in der geriatrischer Physiotherapie“. Ein weiterer wichtiger Schritt für die Qualitätsentwicklung der Arbeit in der Geriatrie seitens der Physiotherapie!

Für den FPG-Vorstand Romy Wälle 


\section{Schweizerische Fachgesellschaft für Gerontopsychologie (SFGP)}

Die Fachgesellschaft der Gerontopsychologie SFGP arbeitete am Themendossier von Curaviva Schweiz zur medizinischen und therapeutischen Versorgung im Alters- und Pflegeinstitutionen mit. Hier eine kurze Zusammenfassung des Beitrags:

Die Gerontopsychologie befasst sich mit dem Erleben und Verhalten von älteren Menschen und wie dieses positiv beeinflusst werden kann. Das gerontopsychologische Leistungsspektrum ist sehr breit und richtet sich nicht nur an die Heimbewohner/innen, sondern auch an deren An- gehörige und an die Institutionsmitarbeitenden. Gerontopsycholog/innen sind heute in Institutionen der Langzeitpflege aber noch wenig präsent. Dies in Zukunft zu ändern, ist wichtig, um im Rahmen der medizinischen und therapeutischen Versorgung optimal und individuell auf die Bedürfnisse eingehen zu können - auf die Bedürfnisse der Heimbewohner/innen (z.B mit einer Demenzerkrankung oder mit Migrationshintergrund), der Angehörigen (z.B. mit Anpassungsschwierigkeiten während oder nach dem Versterben des/der Bewohner/in) und auch der Institutionsmitarbeitenden (z. B. mit hoher Arbeitsbelastung).

Für den SFGP-Vorstand, Regula Bockstaller

\section{Arbeitsgruppe Gerontechnologie}

Im Juni 2012 hat die Schweizer Gesellschaft für Gerontologie eine Arbeitsgruppe ins Leben gerufen, die Personen aus verschiedenen Fachbereichen und Tätigkeitsfeldern vereint und sich mit Gerontechnologie befasst. Diese Gruppe reflektiert die Entwicklung der entsprechenden Technologien und fördert das erforderliche Wissen sowie die benötigten Kompetenzen.

Die Arbeitsgruppe beschäftigt sich mit verschiedenen Forschungsgebieten und praxisorientierten Projekten, wobei ein Projekt grösseren Umfangs auf die folgende Thematik ausgerichtet ist: „Entwicklung, Einsatz und Effektivität der Technologie für die häusliche Betreuung von Senioren“. Hauptziel hierbei ist, die erkannten Bedürfnisse und die Akzeptanz der neu entwickelten Technologien für die häusliche Betreuung von Senioren systematisch zu prüfen, besonders im Hinblick auf altersbedingt gebrechliche Personen bzw. Personen, die ihre Unabhängigkeit/Autonomie verloren haben. Die entsprechende Forschungsarbeit steht kurz vor dem Abschluss. In diesem Zusammenhang kooperiert die Arbeitsgruppe eng mit dem Projekt „Senior Living Lab“, das von einem Gruppenmitglied, Prof. Henk Verloo, betreut wird, sowie mit dem Projekt zur gerontechnologischen Bedarfsermittlung, das vom Verfasser dieses Textes in den Kantonen Genf und Waadt geleitet wird.
Darüber hinaus war die Gruppe in diesem Jahr mit einer weiteren grösseren Angelegenheit beschäftigt: Der Vorbereitung des nationalen SGG-Kongresses „alter@ technik" vom 28./29. Januar 2016 in Freiburg. Hierzu hat die Gruppe bei der Erstellung des Programms mitgewirkt und bekannte Persönlichkeiten auf dem Gebiet der Gerontechnologie eingeladen. Auch wird sie in enger $\mathrm{Zu}$ sammenarbeit mit den wichtigsten Schweizer Universitäten einen Stand betreiben und Projekte zu Technologien und zur Autonomie von Senioren vorstellen, die derzeit in unserem Land durchgeführt werden.

Ferner bemüht sich die Arbeitsgruppe um die Organisation von Thementagen wie z.B. „Gerontechnologie und die Anpassung des Lebensraums an Alter und Gebrechlichkeit".

Der erste solche Tag ist für 2016 vorgesehen; danach wird jeweils einmal im Jahr ein Thementag stattfinden.

Schliesslich ist die Arbeitsgruppe auch intensiv an der Organisation des nächsten internationalen Gerontechnologiekongresses der International Society for Gerontechnology beteiligt, der vom 28. bis 30. September 2016 in Nizza stattfinden wird, sowie an der Vorbereitung des internationalen französischsprachigen Geriatrie- und Gerontologiekongresses (AIFGG), der 2018 in der Schweiz abgehalten wird.

Für die Arbeitsgruppe, Luigi Corrado

\section{Arbeitsgruppe Pflegewohnungen}

Das Jahr 2015 war für die Arbeitsgruppe Pflegewohnungen geprägt durch die Verbreiterung der Mitgliederbasis. In gestärkter Form soll nun der Aufbau einer Website zum Thema Pflegewohnungen angegangen werden. Dazu gehören Klärungen bezüglich Trägerschaft und Finanzierung. Dafür liegen bereits wesentliche Bausteine vor wie z.B. eine Domain-Adresse www.pflegewohnung.ch, der
Gesuchstext mit Finanzplan und ein graphisches Konzept mit einer Sitemap-Struktur.

SGG-Mitglieder mit Erfahrungen und Kompetenzen beim Aufbau und im Betrieb von Pflegewohnungen sind eingeladen, bei Interesse an einer Mitarbeit am Aufbau oder an der Bewirtschaftung, sich bei der SGG-Arbeitsgruppe zu melden:

Kontakte zur SGG-Arbeitsgruppe Pflegewohnungen:

Leiterin: 
Dorothea Schlapbach

Spitalgasse 29 in 3011 Bern

T. 0313111926

Mail: schlapbach@age-network.ch

oder
Matthias Hürlimann

Dipl. Architekt ETH

T. 0442414044

Mail: huerlimann@altervia.ch

\section{Arbeitsgruppe Netzwerk Altersbeauftragte}

Im Jahre 2015 fanden zwei Treffen statt. Das Frühjahrstreffen stand unter dem Thema „Einbezug der Angehörigen von älteren Menschen“. Die Praxis zeigt immer wieder, dass vor allem Angehörige sich erkundigen, was es an Betreuungs- und Versorgungsangeboten für ihre älteren Verwandten gibt. Es braucht eine gemeinsame Lösungssuche aller Beteiligter.

Das Herbsttreffen 2015 widmete sich dem Thema „Netzwerk“. In einem ersten Teil stand die Fortführung der SGG-Arbeitsgruppe zur Diskussion: Was soll das nationale Netzwerk der Altersbeauftragten SGG SSG vermitteln? Im zweiten Teil stellte sich der Verein „Evivo Netzwerk“ vor. Evivo setzt sich mit dem Kurs „Gesund und aktiv leben“ für ein gesundheitsbewusstes und selbstbestimmtes Leben ein. Dieses Self-Management wurde an der Universität Stanford mit Betroffenen und Fachleuten entwickelt und wird in der Schweiz durch die Careum Stiftung getragen.

\section{Zur aktuellen Situation der Arbeitsgruppe}

Bereits seit einiger Zeit werden Sinn und Zweck des SGGNetzwerkes Altersbeauftragte von aktiven Mitgliedern im- mer wieder hinterfragt. Als 1990 die Arbeitsgruppe „Vernetzte Alterspolitik SGG“ gegründet wurde, hatte diese Pionierfunktion wie auch das 2005 daraus entstandene und bis heute existierende Netzwerk. Es gab damals nur wenige Altersbeauftragte, Austausch und Zusammenarbeit auf nationaler oder zumindest deutschschweizerischer Ebene waren gefragt. Mittlerweile ist viel passiert.

Erfreulicherweise gibt es heute viel mehr Altersbeauftragte (nach wie vor mit sehr unterschiedlichen Aufgaben und Kompetenzen). In diesem Zuge haben sich auch regionale Netzwerke gebildet, die den Austausch und die Zusammenarbeit pflegen und deren Mitglieder nicht noch an einem überregionalen Netzwerk teilnehmen möchten. Im Weiteren ist 2012 das Netzwerk der altersfreundlichen Städte gegründet worden, getragen vom Schweizerischen Städteverband. Angesichts all dieser Entwicklungen sollen nun im 2016 Zweck und Aufgaben des „Netzwerks Altersbeauftragte“ überdacht und vom SGGVorstand neu definiert werden. Ziel könnte eine Arbeitsgruppe im engeren Sinne sein, die nach wie vor den Fokus auf der Gemeindeebene hat und sich spezifischer und definierter Aufgaben annehmen wird.

Für die Arbeitsgruppe „Netzwerk Altersbeauftragte“, Hansjürg Rohner

\section{Arbeitsgruppe Ethik und Spiritualität}

Vielleicht kennen Sie die eine oder andere Publikation der Arbeitsgruppe „Ethik und Spiritualität“: 1999 erschien das Praxishandbuch „Altersarbeit: Leben gestalten - Kirche leben“, 2007 die Handreichung „Die spirituelle Dimension braucht Raum“ und im Frühjahr 2015 die Broschüre „Ethische Entscheidfindung in der ambulanten und stationären Langzeitpflege“. Die beiden letzten Publikationen richten sich vorab an Mitarbeitende in der Langzeitpflege, an Angehörige von alten Menschen in Abhängigkeit und an Freiwillige, die alte, auf Unterstützung angewiesene Menschen begleiten.

Verschiedentlich haben wir bereits $\mathrm{zu}$ Themen von Ethik und Spiritualität im Auftrag des SGG-Vorstandes Stellungnahmen erarbeit, so beispielsweise zur Gesetzesänderung betreffend organisierter Suizidhilfe, zu den SAMW-Empfehlungen „Strukturen zur ethischen Unterstützung in der Medizin" oder zu den SAMW-Richtlinien „Intensivmedizinische Massnahmen“. Gestützt auf Vorar- beiten der Arbeitsgruppe hat der SGG-Vorstand auch das Positionspapier „Suizidbeihilfe für alte Menschen“ publiziert. Neue Aufgaben hat unsere Arbeitsgruppe im Umsetzungsprojekt 5.1. der Nationalen Demenzstrategie „Verankerung ethischer Leitlinien“, für das die SGG zusammen mit der SAMW den Lead übernommen hat.

Um diese wichtigen Aufgaben für die SGG auch weiterhin erfolgreich erfüllen zu können, freuen wir uns über neue Mitglieder und Unterstützung unserer AG. Interessieren Sie sich für ethische Fragestellungen? Sind Ihnen spirituelle Bedürfnisse alter Menschen ein Anliegen? Wenn Sie an einer Mitarbeit in der Arbeitsgruppe Ethik und Spiritualität interessiert sind, so melden Sie sich doch bei einer der Co-Leiterinnen, wir freuen uns über Ihre Mitarbeit!

Esther Enderli

e-mail: esther.enderli@bluewin.ch

Simone Anna Heitlinger:

e-mail: s.heitlinger@bluewin.ch oder simone.heitlinger@ spitalaffoltern.ch 


\section{SFGG.SPSG}

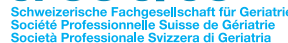

Zunächst einmal gratuliert die Schweizerische Fachgesellschaft für Geriatrie ihrer Schwestergesellschaft zur neuen Zeitschrift. Wir sind der festen Überzeugung, dass unter dem Titel „Angewandte Gerontologie“ eine Lücke geschlossen wurde, die gerontologische Inhalte aus der Praxis für die Praxis vermitteln soll. Damit hat die SFGG auch eine neue Plattform erhalten, um einer interessierten Leserschaft über die Aktivitäten der SFGG zu berichten. Die SFGG wünscht dem Redaktionsteam einen guten Start und dass stets Gut zum Druck vorhanden ist. Gerne fordere ich auch die SFGG Mitglieder auf, Beträge einzureichen.

\section{Internationaler Frankofoner Kongress}

Unsere Fachgesellschaft freut sich, den Internationalen Frankofonen Kongress für Geriatrie und Gerontologie mitorganisieren zu dürfen, der 2018 in Montreux stattfinden wird. Hier sind unsere Kolleginnen und Kollegen aus der Westschweiz besonders gefordert. Erste Vorbereitungsgespräche sind erfolgreich geführt worden.

\section{SGAP SPPA}

\section{Schweizerische Gesellschaft für Alterspsychiatrie und -psychotherapie (SGAP)}

\section{Erfolgreicher Internationaler Sommerkurs}

Zum zweiten Mal bereits organisierte die Klinik für Alterspsychiatrie der Universität Lausanne (SUPAA; Service Universitaire de Psychiatrie de l'Âge Avancé) einen einwöchigen internationalen Sommerkurs für junge Psychiaterinnen und Psychiater, die sich im Altersbereich spezialisiert haben. Der Kurs fand unter der Schirmherrschaft der europäischen Vereinigung für Alterspsychiatrie (European Association of Geriatric Psychiatry - EAGP) statt. Nebst den hochkarätigen Fachbeiträgen setzten sich die Organisatoren zum Ziel, einen Grenzen übergreifenden Austausch zu ermöglichen. Die jungen Kollegen aus Deutschland, Frankreich und der Schweiz profitierten von namhaften Referenten und Workshop-Leitern, die ebenfalls den Weg nach Lausanne machten: Profs F. Boukaert, V. Camus, J. Ghika, R. Ihl, U. Mosimann, R. Oude Voshaar, P. Robert, M. Orrell, R. Tomar, B. van Alphen und F. Ver-

\section{Nationale Demenzstrategie}

Unsere Fachgesellschaft übernimmt gemeinsam mit Ihrer Partnergesellschaft, der Schweizerischen Gesellschaft für Alterspsychiatrie und -psychotherapie (SGAP), den Lead in der Umsetzung des Projektes 6.2 (Interdisziplinäre Assessments) der Nationalen Demenzstrategie. Auch hier haben erste vorbereitende Sitzungen stattgefunden. Ansprechpartner sind Gaby Bieri (SFGG) und Dan Georgescu (SGAP). An der Mitarbeit interessierte Kolleginnen und Kollegen wenden sich bitte an die Geschäftsstelle.

\section{Workshop Verbesserung der koordinierten Versorgung (hoch)betagter multimorbider Menschen}

Am 13.11. fand der 2. Workshop zu dieser Thematik im BAG in Bern im Rahmen der Agenda "Gesundheit 2020“ unter Mitarbeit der SFGG statt. In einer sehr konstruktiven Atmosphäre wurden Ergebnisse des ersten Workshops im September überarbeitet. Die Diskussion konzentrierte sich auf unmittelbar umsetzbare Massnahmen. Es zeigte sich, dass vor allem im Akutbereich ein Mangel an geriatrischer, gerontopsychiatrischer und gerontologischer Expertise herrscht. Hier ist das BAG aufgefordert, entsprechende Massnahmen im Bildungsbereich und/oder der Ausbildung von Pflegepersonen und Ärzten in die Wege zu leiten.

Thomas Münzer, Präsident

hey. Der Kurs wurde von den Teilnehmern als qualitativ hochstehend bewertet. Alle waren der Meinung, dass er erneut und in regelmässigen Abständen organisiert werden müsste. Dementsprechend ist im Sommer 2017 in Lausanne ein dritter Sommerkurs (summer school) bereits geplant.

Für das Präsidium, Armin von Gunten

\section{5-jähriges Jubiläum der SGAP}

Dieses Jahr feiert die SGAP ihr 25-jähriges Jubiläum! Die Mitgliederversammlung der SGAP vom 16.06.2016 wird dementsprechend inklusive Mittagessen und abschliessendem Fest-Apéro in der Cinematte Bern stattfinden. Geplant ist ebenfalls ein Jubiläumssymposium am SGPP Kongress 2016 (Mi 17.08. - Fr 19.08.2016).

Alterspsychiatrie ist die Psychiatrie für ältere Menschen, wobei diese Definition so sicher nicht genügt. Wir behandeln Menschen mit psychischen Krankheiten, welche schon seit vielen Jahren bestehen (Schizophrenien, Sucht), doch vor allem kümmern wir uns um alterstypische Krankheitsbilder (Depression, Demenz und Delir), die auf spezifische Art diagnostiziert und behandelt werden müssen. Das Bewusstsein der Möglichkeit oder sogar Notwendigkeit, etwas für die ärztliche Unterstützung alter Menschen mit psychischen Störungen zu unternehmen, ist noch gar nicht so alt. 
Zur Zeit der Gründung der SGAP gingen nur wenige ältere Menschen freiwillig zum Psychiater, nicht zuletzt darum, weil diese auch eigene Berührungsängste hatten, besonders zu somatischen Fragen. Die Anzahl älterer Menschen nimmt stetig zu und ihre Ansprüche verändern sich: Sie erwarten mittlerweile qualitativ hoch stehende psychiatrische Dienstleistungen - auch für ihre Altersgruppe. Der/die heu- tige Alterspsychiater/in muss in Sachen Fachkompetenz stets auf dem neuesten Stand sein. Dieser professionelle Ansatz war dann auch ausschlaggebend für die Geburt unserer Fachgesellschaft vor 25 Jahren.

Für den SGAP-Vorstand, Daniel Strub

\section{Au revoir et merci}

Chers membres de la SSG,

Mon mandat se termine avec le congrès national de la SGG-SSG 2016. Je tiens, chers membres, à vous remercier chaleureusement pour votre soutien de la SSG dans le cadre de vos activités professionnelles et volontaires au cours de mon mandat et, ainsi que je l'espère, au-delà aussi. Sans votre contribution, que ce soit dans le domaine scientifique ou celui de la gérontologie appliquée, la SSG ne pourrait agir et contribuer à l'amélioration de la situation de vie des personnes âgées au sein de notre société, aujourd'hui et demain!

Je vous remercie aussi de votre confiance qui m'a permis pendant quatre ans de m'engager activement pour les objectifs de la SSG et les propos de la gérontologie. Ce fut une période intensive et très passionnante, qui m'a permis de faire de nombreuses rencontres personnelles dépassant le cadre de la pure fonction. Elles perdureront certainement et enrichiront ma vie en tant que gérontologue tout comme en tant que personne privée.

Mes remerciements vont plus particulièrement aux membres de nos divers groupes spécialisés et groupes de travail pour leur engagement volontaire : le Groupe de professionnels en gérontologie appliquée (GPGA) et ses forums régionaux, le Groupe spécialisé de physiothérapie en gériatrie (GPG) et ses formations continues toujours bien suivies, ainsi que la Société professionnelle suisse de Gérontopsychologie (SPGP) qui s'engage pour des possibilités de traitements adaptés à l'âge. La contribution apportée par nos groupes de travail est également importante : le groupe de travail Éthique et spiritualité, qui a élaboré la brochure très appréciée « Prise de décision éthique dans les soins de longue durée ambulants et stationnaires », le groupe Gérontechnologies, activement impliqué dans les préparations et la conception du congrès de 2016, ainsi que le groupe de travail Appartements médicalisés, qui se mobilise pour de nouvelles formes de prise en charge.

Un tout grand merci aussi à tous les membres du comité de la SSG pour la collaboration personnellement très agréable et surtout constructive. Vos questions et vos contributions aux discussions m'ont toujours stimulée et parfois même interpellée. Elles ont en tout cas énormément favorisé le développement de la SSG. Et last but not least, tout mon engagement pour la SSG n'aurait pas été possible sans l'appui extrêmement compétent et constructif de notre secrétariat, en la personne de Monika Steiger et Beatrice Böhlen.

Un grand merci à toutes et à tous; grâce à vous j'ai pu vivre quatre années de présidence passionnantes et nous avons pu atteindre beaucoup ensemble !

Mon adieu n'est toutefois qu'un «demi», car c'est avec plaisir que je continuerai à m'engager pour la SSG en tant que past-présidente et que je resterai ainsi en contact étroit avec elle. Je souhaite aujourd'hui déjà bonne continuation et plein succès à la nouvelle présidence ainsi qu'au comité et surtout autant de satisfaction que celle que j'ai pu éprouver!

\section{Cordialement}

Votre

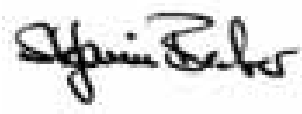




\section{Groupe des professionnels en gérontologie appliquée (GPGA)}

\section{La voie est libre pour un quatrième forum GPGA de la gérontologie appliquée !}

Lors de sa réunion de septembre, le comité du groupe spécialisé « Groupe des professionnels en gérontologie appliquée (GPGA) » a pris acte avec plaisir des plans pour un quatrième forum régional de la gérontologie appliquée. Le noyau impliqué dans le nouveau forum comprend déjà quatre personnes pour la région Suisse septentrionale. Tous les membres GPGA de la région seront également contactés personnellement.

Les prochaines dates des forums de gérontologie appliquée GPGA à Berne, Lucerne et Zurich sont déjà fixés :

Forum de gérontologie appliquée pour la région de Zurich/Suisse orientale

- Mercredi 1.6.2016,18h00

- Jeudi 29.9.2016,18h00
Forum de gérontologie appliquée pour la région de Berne

- Lundi, 22.2.2016, 16h30

- Lundi, 13.6.2016, 16h30

- Lundi ,24.10.2016, 16h30

Forum de gérontologie appliquée pour la région de $\mathrm{Zu}$ rich/Suisse centrale

- Jeudi, 10.3.2016,17h00

- Jeudi, 12.5.2016,17h00

- Jeudi, 15.9.2016, 17h00

- Jeudi, 1.12.2016, 17h00

NOUVEAU : Forum de gérontologie appliquée pour la région de la Suisse du Nord-Ouest (les deux Bâle, Aargau, Solothurn)

Les informations seront publiées sur www.sgg-ssg.ch/ GPGA

Vous trouverez des informations sur les différents thèmes prévus et le lieu de la rencontre sur www.sgg-ssg.ch (GPGA). Pour le comité du GPGA, Stefan Spring

\section{Groupe spécialisé de physiothérapie en gériatrie (GPG)}

\section{Des images en disent bien plus long que des mots}

Le GPG a atteint un de ses grands objectifs pour 2015 : la bibliothèque de photos est terminée. Plus de 100 photos montrent de manière impressionnante ce que nous pouvons faire bouger en tant que physiothérapeutes. Une relation de confiance entre patient/patiente et thérapeute est indispensable pour une thérapie réussie. Le photographe Bruno Wyss est parvenu à la capter, ainsi que bien d'autres choses. Les photos pourront bientôt être découvertes sur la site internet de la SSG ( $>$ Groupes spécialisés $>$ GPG). Ma- dame Christine Frey-Widmer, physio. frey@gmx.ch, (tél. G : 043497 81 17) est l'interlocutrice chez GPG pour l'utilisation de ces photos. Vous pouvez vous adresser à elle à tout moment.

Un autre jalon a été atteint dans la collaboration avec la Fachhochschule Gesundheit de Winterthur (ZHAW). À partir de 2017, un CAS modulaire pourra être suivi en physiothérapie gériatrique. Cette nouvelle offre sera présentée plus en détail le 27 août 2016, dans le cadre d'un symposium sur le thème « Nouvelles voies dans la physiothérapie gériatrique ». Une autre étape importante pour le développement de la qualité du travail en physiothérapie gériatrique!

Pour le comité du GPG, Romy Wälle

\section{Société professionnelle suisse de gérontopsychologie (SPGP)}

La Société professionnelle suisse de gérontopsychologie, SPGP, travaille sur le dossier thématique de Curaviva Suisse concernant la prise en charge médicale et thérapeutique dans les EMS et homes pour personnes âgées. Voici un bref résumé de la contribution:

La gérontopsychologie s'attache au vécu et au comportement des personnes âgées ainsi qu'à la manière dont ceux-ci peuvent être influencés positivement. Le spectre des services gérontopsychologiques est très large et ne s'adresse pas seulement aux habitants des homes et insti- tutions, mais aussi à leurs familles ainsi qu'aux collaborateurs des institutions. Les gérontopsychologues sont toutefois encore peu présents dans les institutions de soins de longue durée. Changer cette situation est important, afin de pouvoir répondre aux besoins dans le cadre d'une prise en charge médicale et thérapeutique optimale et individuelle, qu'il s'agisse des besoins des habitants et habitantes du home (p. ex. souffrant de démence ou issues de la migration), de la famille (p. ex. avec difficultés d'adaptation pendant ou après le décès de leur proche) ou des collaborateurs et collaboratrices de l'institution (p. ex. avec charge de travail élevée).

Pour le comité de la SPGP, Regula Bockstaller 


\section{Groupe de travail Gérontechnologies}

En juin 2012, la Société Suisse de Gérontologie a créé un groupe de travail réunissant des personnes de diverses disciplines et champs d'activité dédié à la Gérontechnologie pour mener une réflexion, sur l'évolution de ces technologies, et développer aussi les savoirs nécessaires et les compétences requises.

Ce groupe de travail s'intéresse à différentes recherches et projets orientés sur la pratique, dont un majeur axé sur la thématique : «Émergence, utilisation et efficacité de la technologie pour le maintien à domicile des personnes âgées », dont l'objectif principal est de mener un examen systématique de la documentation sur les besoins perçus et l'acceptabilité des technologies émergentes pour le maintien à domicile des personnes âgées, et plus particulièrement les personnes confrontées à une fragilité du fait de leur âge ou en perte d'indépendance/ d'autonomie. Ce travail de recherche est à bout touchant. Dans ce cadre, le groupe de travail est en lien étroit avec le projet Senior $\mathrm{Li}$ ving Lab conduit par un de ses membres, le Pr. Henk Verloo, ainsi que le projet d'enquête des besoins en gérontechnologies mené par le soussigné dans les cantons de Genève et Vaud.
Un autre dossier majeur a occupé le groupe de travail durant cette année. La préparation de notre congrès de janvier prochain à Fribourg âge@technique. En effet, outre le fait d'avoir contribué à la préparation du programme et invité des personnalités connues dans le domaine de la gérontechnologie, le groupe de travail tiendra un stand, en étroite collaboration avec les principales Universités de Suisse, et présentera les projets en cours dans notre pays en lien avec les technologies et l'autonomie des personnes âgées.

Le groupe de travail a pour ambition également d'organiser des journées thématiques telles que « l'habitat adapté à la vieillesse et à la fragilité en lien avec la gérontechnologie ».

Celles-ci débuteront en 2016 et se poursuivront au rythme d'une journée par année.

Le groupe de travail est aussi fortement impliqué dans l'organisation du prochain congrès international de gérontechnologie, orchestré par l'International Society for Gerontechnology, qui se déroulera à Nice du 28 au 30 septembre 2016, ainsi que dans la préparation du congrès international francophone de gériatrie et de gérontologie (AIFGG) prévu en Suisse en 2018.

Pour le groupe de travail, Luigi Corrado

\section{Groupe de travail Appartements médicalisés}

Pour le groupe de travail Appartements médicalisés, l'année 2015 fut caractérisée par un étoffement du nombre de ses membres, destiné à offrir la base en personnel et en compétence permettant de construire un site Web sur le thème des appartements médicalisés. Il est prévu de fonder une association et une demande de financement déjà préparée sera déposée à cet effet. Les principaux éléments constitutifs existent déjà, p. ex. une adresse de domaine (www.pflegewohnung.ch), le texte de la demande avec plan financier ainsi qu'un concept graphique avec la structure du site.

Les membres de la SSG possédant de l'expérience et des compétences dans la mise en place et le fonctionnement d'appartements médicalisés et intéressés par une collaboration dans le cadre de la mise en place ou du fonctionnement sont priés de contacter le groupe de travail de la SSG :
Contact pour le groupe de travail de la SSG appartements médicalisés :

Responsable :

Dorothea Schlapbach

Spitalgasse 29-3011 Berne

T. 0313111926

Mail : schlapbach@age-network.ch

ou

Matthias Hürlimann

Architecte dipl. FSH

T. 0442414044

Mail : huerlimann@altervia.ch

Pour le groupe de travail, Matthias Hürliimann

\section{Groupe de travail Réseau des coordinateurs pour personnes âgées}

Deux rencontres ont été organisées en 2015. Celle du printemps était consacrée au thème «Implication des proches des personnes âgées ». La pratique montre régulièrement que ce sont surtout les proches qui demandent ce qui existe comme offre de prise en charge et de soins de leurs parents plus âgés. Nous avons besoin d'une solution commune pour toutes les personnes impliquées.

La rencontre d'automne 2015 s'est attachée au thème $\mathrm{du}$ « réseau ». Dans une première partie, nous avons dis- 
cuté d'une prolongation du groupe de travail SSG : qu'estce que le réseau des coordinateurs pour personnes âgées SGG-SSG doit transmettre ? La deuxième partie fut consacrée à la présentation de l'association « Réseau Evivo ». Evivo s'engage pour une vie saine et indépendante avec le cours «Devenir acteur de sa santé». Ce programme d'autogestion a été développé à l'université de Stanford avec des personnes concernées et des spécialistes et est supporté en Suisse par la Fondation Careum.

\section{Situation actuelle du groupe de travail}

Depuis un certain temps déjà, des membres actifs remettent en question le sens et le but du réseau SSG des coordinateurs pour personnes âgées. Lors de la fondation du groupe de travail «Politique de vieillesse en réseau SSG » en 1990, ce groupe faisait fonction de pionnier tout comme le réseau actuel qui en est issu en 2005. À cette époque il n'existait que peu de coordinateurs pour per- sonnes âgées, un échange et une collaboration sur le plan national, ou du moins de la Suisse alémanique, était demandé. Il s'est passé beaucoup de choses entretemps.

Aujourd'hui, nous disposons, heureusement, de plus de coordinateurs pour personnes âgées (même si encore toujours avec des tâches et compétences différentes). Des réseaux régionaux d'échange et de collaboration se sont ainsi formés et leurs membres ne désirent pas nécessairement participer en plus à un réseau supra-régional. À cela s'ajoute la fondation en 2012 du Réseau Suisse des villesamies des aînés supporté par l'Union des villes suisses. Au vu de ces développements, le sens et le but du « Réseau SSG des coordinateurs pour personnes âgées » doivent être revus et redéfinis par le comité de la SSG en 2016. L'objectif pourrait être un groupe de travail au sens étroit du terme, qui se concentrerait comme toujours sur la commune et qui s'attacherait à des tâches spécifiques et définies.

Pour le groupe de travail, Hansjürg Rohner

\section{Groupe de travail Ethique et spiritualité}

Peut-être connaissez-vous l'une ou l'autre publication du groupe de travail « Éthique et spiritualité » ?

Le guide pratique «Altersarbeit : Leben gestalten Kirche Leben » (Organiser son existence, vivre l'Eglise) a été publié en 1999, le guide « Die spirituelle Dimension braucht Raum » (Pour un espace-temps de la dimension spirituelle) en 2007 et la brochure « Ethische Entscheidfindung in der ambulanten und stationären Langzeitpflege » (Prise de décision éthique dans les soins de longue durée ambulants et stationnaires) est parue au printemps 2015. Les deux dernières publications s'adressent principalement aux collaborateurs dans les soins de longue durée, aux membres de la famille des personnes âgées et aux volontaires accompagnant les personnes âgées dépendantes.

Nous avons déjà pris position sur différents thèmes de l'éthique et de la spiritualité au nom du comité de la SSG, entre autres sur l'amendement à la loi sur le suicide assisté, les recommandations de l'ASSM « Structures de soutien éthique de la médecine » ou les directives de l'ASSM «Mesures de de soins intensifs ». Soutenu par les travaux pré- paratoires du groupe de travail, le comité de la SSG a également publié la prise de position «Assistance au suicide pour les personnes âgées ». Notre groupe de travail s'est à présent attelé à de nouvelles tâches dans le cadre du projet de mise en œuvre 5.1 de la stratégie nationale en matière de démence « Ancrage de directives éthiques », dont l'élaboration a été confiée conjointement à la SSG et l'ASSM.

Pour pouvoir continuer à accomplir avec succès ces tâches importantes pour la SSG, nous nous réjouissons de tout nouveau membre et du soutien de notre groupe de travail. Les questions éthiques vous intéressent ? Les besoins spirituels des personnes âgées vous préoccupent? $\mathrm{N}$ 'hésitez pas à contacter une des co-coordinatrices si une participation au groupe de travail Éthique et spiritualité vous intéresse. Nous nous réjouissons de votre collaboration!

Esther Enderli

E-mail : esther.enderli@bluewin.ch

Simone Anna Heitlinger :

e-mail : s.heitlinger@bluewin.ch ou simone.heitlinger@ spitalaffoltern.ch

\section{SFGG.SPSG}

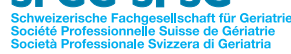

La Société Professionnelle Suisse de Gériatrie tient d'abord à féliciter son association sœur à l'occasion de la sortie de sa nouvelle revue. Nous sommes persuadés que cette revue intitulée «Gérontologie appliquée » et destinée à la transmission de contenus gérontologiques de la pratique pour la pratique vient combler un vide. Avec elle, la SPSG dispose aussi d'un nouvel organe de publication offrant les dernières informations sur les activités de la SPSG au public intéressé. La SPSG souhaite un bon départ à l'équipe de rédaction et toujours un bon à tirer. J'invite les membres de la SPSG à rentrer des articles. 


\section{Congrès international francophone de gériatrie et gérontologie}

Notre société professionnelle se réjouit de participer à l'organisation du Congrès international francophone de gériatrie et gérontologie qui aura lieu à Montreux en 2018. Nos collègues en Romandie seront plus particulièrement sollicités dans ce cadre. Les premiers entretiens préparatoires ont été menés avec succès.

\section{Stratégie nationale en matière de démence}

Avec son association sœur, la Société Suisse de Psychiatrie et Psychothérapie de la Personne Agée (SPPA), notre société professionnelle pilote la mise en œuvre du projet 6.2 (Promotion des évaluations interdisciplinaires) de la stratégie nationale en matière de démence. Des entretiens préparatoires ont déjà été menés ici aussi. Nos interlocuteurs sont Gaby Bieri (SPSG) et Dan Georgescu (SPPA).
J'invite les collègues intéressés par une collaboration à s'adresser au secrétariat.

\section{Atelier " Amélioration de la prise en charge coordonnée de personnes (très) âgées multimorbides"}

La SPSG a collaboré au 2e atelier de l'OFSP à Berne sur ce thème dans le cadre de l'agenda « Santé 2020 ». Les résultats du premier atelier en septembre ont été retravaillés dans une atmosphère très constructive. La discussion s'est à présent concentrée sur des mesures directement réalisables. Il s'avère qu'il existe un manque d'expertise gériatrique, gérontopsychiatrique et gérontologique surtout dans le domaine de la prise en charge aigüe. L'OFSP doit mettre en place les mesures correspondantes dans le domaine de l'éducation et/ou de la formation du personnel soignant et des médecins.

Thomas Münzer, président

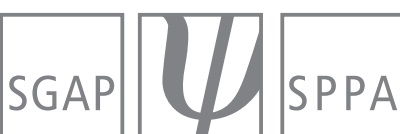

\section{Société Suisse de Psychiatrie et Psychothérapie de la Personne Agée (SPPA)}

\section{Succès du cours d'été international}

Pour la deuxième fois déjà, le Service Universitaire de Psychiatrie de l'Âge Avancé (SUPAA) de l'Université de Lausanne a organisé un cours d'été international d'une semaine pour les jeunes psychiatres désirant se spécialiser dans le domaine des personnes âgées. Le cours s'est déroulé sous les auspices de l'European Association of Geriatric Psychiatry - EAGP. À côté des contributions spécialisées de haut niveau, l'objectif des organisateurs était de permettre un échange transfrontalier. Les jeunes collègues venus d'Allemagne, de France et de la Suisse ont pu profiter de présentations données par des orateurs de renom et de responsables d'ateliers ayant également rejoint Lausanne : les professeurs F. Boukaert, V. Camus, J. Ghika, R. Ihl, U. Mosimann, R. Oude Voshaar, P. Robert, M. Orrell, R. Tomar, B. van Alphen et F. Verhey. Les participants ont unanimement évalué le cours comme offrant un haut niveau de qualité. Ils étaient tous d'avis qu'il devrait être réorganisé à des intervalles réguliers. C'est pourquoi un troisième cours d'été (summer school) est déjà prévu à Lausanne en été 2017.

Pour la présidence, Armin von Gunten

\section{5e jubilé de la SPPA}

La SPPA fêtera son $25^{\mathrm{e}}$ anniversaire en 2016 ! L'assemblée générale de la SPPA du 16.06.2016 se déroulera donc dans la Cinematte Berne, avec un repas de midi et un apéro festif de conclusion. Un symposium jubilaire est également prévu à l'occasion du Congrès 2016 de la SSPP (me 17.08 au ve 19.08.2016).

La psychiatrie de la personne âgée est destinée aux aînés : en tant que telle, cette définition ne suffit certainement pas. Nous traitons les personnes atteintes de maladies psychiques depuis de nombreuses années (schizophrénie, dépendances), mais nous nous occupons surtout des maladies typiquement liées à l'âge (dépression, démence et délire) qui doivent être diagnostiquées et traitées de manière spécifique.

Il n'y a pas si longtemps que l'on a pris conscience des possibilités voire de la nécessité de faire quelque chose pour soutenir médicalement les personnes âgées présentant des troubles psychiques. Au moment de la fondation de la SPPA, seules quelques rares personnes âgées consultaient volontairement un psychiatre, partiellement aussi parce qu'eux-mêmes avaient leurs propres peurs, particulièrement en ce qui concerne les questions somatiques. Le nombre de personnes âgées augmente constamment et leurs exigences changent : elles attendent maintenant des services psychiatriques de haute qualité - pour leur groupe d`âge aussi. Le/la psychiatre d'aujourd'hui doit toujours être au courant des dernières avancées en termes de compétence. Cete approche professionnelle a donc donné lieu à la naissance de notre société professionnelle il y a 25 ans. 


\section{Adressen der Gesellschaften:}

Schweizerische Gesellschaft für Gerontologie SGG SSG Geschäftsstelle

Haus der Akademien

Postfach

CH-3001 Bern

info@sgg-ssg.ch

www.sgg-ssg.ch

Schweizerische Fachgesellschaft für Geriatrie SFGG SPSG

Geschäftsstelle

Haus der Akademien

Postfach

CH-3001 Bern

info@sfgg.ch

www.sfgg.ch

Schweizerische Gesellschaft für Alterspsychiatrie und -psychotherapie SGAP SPPA

Sekretariat

Postfach 698

3000 Bern 8

info@sgap-sppa.ch

www.sgap-sppa.ch

\section{Adresses des sociétés:}

Société Suisse de Gérontologie SGG SSG

Secrétariat

Maison des Académies

Case postale

CH-3001 Berne

info@sgg-ssg.ch

www.sgg-ssg.ch

Société Professionnelle Suisse de Gériatrie SFGG SPSG

Secrétariat

Maison des Académies

Case postale

CH-3001 Berne

info@sfgg.ch

www.sfgg.ch

Société Suisse de Psychiatrie et Psychothérapie de la Personne Agée SGAP SPPA

Secrétariat

Case postale 686

3000 Berne

info@sgap-sppa.ch

www.sgap-sppa.ch 


\section{Aktuelle Übersicht zum Schweizer Gesundheitswesen}
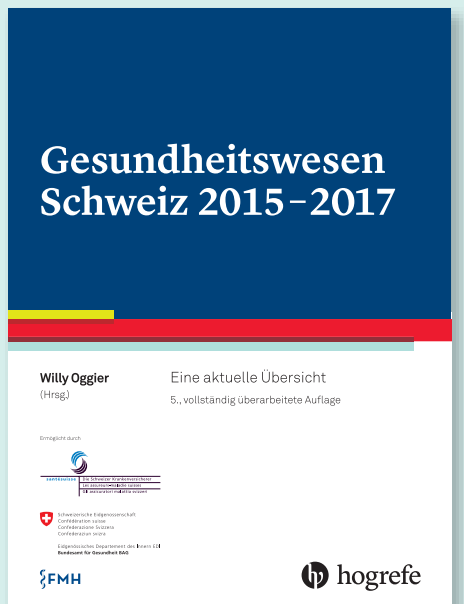

Willy Oggier (Hrsg.)

\section{Gesundheitswesen Schweiz 2015-2017}

Eine aktuelle Übersicht

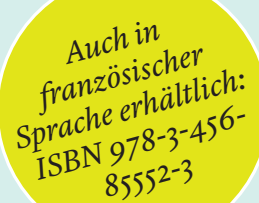

5., vollst. überarb. Aufl. 2015.

488 S., 85 Abb., 129 Tab., Gb

$€ 29.95 /$ CHF 39.90

ISBN 978-3-456-85441-0

AUCH ALS E-BOOK

Das Schweizer Gesundheitswesen ist komplex und im Umbruch. Reformversuche und Sparmassnahmen jagen sich, oft ohne klare Ziele und ohne Koordination. Gesundheitspolitik ist seit Jahren ein heissumstrittener und komplexer Politikbereich - mit ideologischen Polemiken und starken Interessengruppen. In einer solchen Lage ist Transparenz wichtig.

Dieses Buch ist das Standardwerk über das schweizerische Gesundheitswesen. Es gibt eine aktuelle und sachliche Gesamtübersicht in 39 Kapiteln von $61 \mathrm{Au}$ torinnen und Autoren, wobei besonderer Wert gelegt wurde auf möglichst hohe Objektivität, aussagekräftige Daten und auf gute Lesbarkeit. Gegenüber der 4 Auflage 2010 wurde es vollständig überarbeitet, erweitert und aktualisiert.

Das Buch ist ein Nachschlagewerk für alle, die sich für das Gesundheitssystem und die Gesundheitspolitik interessieren: Fachleute aus dem Gesundheitswesen und den Sozialversicherungen, für Politik, Medien und Wissenschaft und selbstverständlich auch für interessierte Versicherte und Patientinnen/Patienten. Besonders geeignet ist es auch als Lehrmittel in den verschiedenen Aus-, Weiter- und Fortbildungsgängen im Gesundheits-, Sozial- und Versicherungswesen. 